\title{
Young pregnant woman with mild dysphagia: Diagnostic and treatment workup predicting laryngeal schwannoma
}

\author{
Martelli F $F^{1}$, Squadrelli $M^{2}$, Santoro $R^{2}$, Spinato $G^{3,4}$ and Mannelli G $^{2 *}$ \\ ${ }^{1}$ University of Florence, Italy \\ ${ }^{2}$ Head and Neck Oncology and Robotic Surgery, Department of Experimental and Clinical Medicine, University of Florence, Azienda Ospedaliero Universitaria \\ Careggi, Largo Palagi 1, 50134, Florence, Italy \\ ${ }^{3}$ Department of Neurosciences, Section of Otolaryngology and Regional Centre for Head and Neck Cancer, University of Padova, Treviso, Italy \\ ${ }^{4}$ Department of Surgery, Oncology and Gastroenterology, Section of Oncology and Immunology, University of Padova, Padova, Italy
}

\begin{abstract}
Schwannomas of the larynx are uncommon, they represent the $0.1-1.5 \%$ of all the benign laryngeal tumours. Patients usually report multiple symptoms related to mass-effects and their treatment of choice is surgical excision. We present the case of a young woman presenting a schwannoma within her left false vocal fold, not suitable for endoscopic surgical approach. The suspected diagnosis of schwannoma was confirmed by the final histopathological report, showing a protein S110 positivity at the immunohistochemistry staining. Complete surgical excision is the treatment of choice and can be performed by either transoral or external approach depending on lesion dimension, patient's laryngeal exposure and surgeon's skills. In both cases we do suggest a conservative approach.
\end{abstract}

\section{Introduction}

Schwannomas, also known as neurinomas, are benign peripheral nerve sheath tumours arising from the Schwann cells of any nerve (peripheral, cranial, or autonomic). A range of $25-45 \%$ occurs in the head and neck, and the majority of them can be found intracranial or in the parapharyngeal space [1]. Schwannomas originating within the larynx are instead uncommon, representing less than $1.5 \%$ of all the benign laryngeal tumours [2]. They are thought to arise from the internal branch of the superior laryngeal nerve and the vast majority of the reported cases are supraglottic, affecting the false vocal cords or the aryepiglottic fold [3]. They are slow-growing neoplasms that typically appear on laryngoscopy as round submucosal swelling and patients usually report multiple symptoms related to mass-effects like dysphagia, odynophagia, hoarseness or dysphonia, globes sensation and dyspnea. Appropriate treatment requires the complete surgical excision, both with endoscopic or open approaches [4]. We describe the case of a young woman presenting a schwannoma within her left false vocal fold, not suitable for endoscopic surgical approach.

\section{Case report}

In February 2016, a 32-year-old pregnant woman, was referred to our Institution, for the evaluation of pharyngodynia and odynophagia not responding to conventional medications. She denied other symptoms as dispnoea, otalgia or recent respiratory tract infection and there was not associated systemic symptoms. She was a non-smoker and non-alcohol consumer. Flexible laryngoscopy revealed the presence of a left false vocal cord submucosal mass, extended to the aryepiglottic fold, with maximum diameter of $2 \mathrm{~cm}$, causing a mild obstruction of the laryngeal inlet. Both vocal folds were mobile and no cervical lymph nodes were palpable. Due to pregnancy, young age and the absence of exposure to cancer risk factors (e.g. smoke), we decided to proceed with a close clinical follow-up, with a primary suspected diagnosis of laryngocele. During this time, no dimensional increase of the mass or aggravation of symptoms were observed.

Magnetic resonance imaging (MRI) scan was scheduled immediately after the delivery. The radiological exam showed the presence of a capsulated oval mass, $2 \mathrm{~cm}$ in its maximum diameter, with regular margins, hypointense on T1 and hyperintense on T2 and STIR weighted images with heterogeneous enhancement after gadolinium. Radiological findings were suspicious for either a laryngocele or schwannoma (Figure 1)

Because of the small dimension of the mass, a transoral microlaryngoscopy procedure was planned but due to an unexpected bad laryngeal exposure an open surgical approach was proposed.

During prior assessment by using clinical predictor score for difficult laryngeal exposure, the patient was classified as Class 0 in according to Laryngoscore, with complete visualization of the anterior commissure during flexible laryngoscopy. No trismus, mandibular prognathism, macroglossia or micrognathia was revealed and the only adverse feature observed was a Mallampati class of grade I-II [5].

At the time of open approach, a horizontal skin incision of $4 \mathrm{~cm}$ was made at the level of the superior border of the left thyroid cartilage lamina. Subplatysmal flap was raised and proceeding in depth the ipsilateral strap muscle where sectioned to obtain good exposure of

${ }^{*}$ Correspondence to: Giuditta Mannelli, MD, Head and Neck Oncology and Robotic Surgery, Department of Experimental and Clinical Medicine, University of Florence, AOU-Careggi, Largo Palagi 1, 50134, Florence, Italy, E-mail: giuditta.mannelli@unifi.it

Key words: pregnancy, dysphagia, workup, laryngeal tumour, schwannoma

Received: October 02, 2019; Accepted: October 23, 2019; Published: October 28,2019 
the surgical field. Thyrohyoid membrane was exposed and incised to access the superior paraglottic space. The thyroid lamina on the affected side were retracted with a hook and the mass was then removed by blunt dissection taking care at maintaining the integrity of the overlying laryngeal mucosa and preserving the trunk of the internal branch of the superior laryngeal nerve. Strap muscles were sutured, and a pen-rose drain was placed to prevent supraglottic hematoma. The respiratory space was checked by direct laryngoscopy at the end of the procedure with no evidence of oedema impairing laryngeal airway. No tracheotomy was necessary (Figure 2).

The surgical specimen was a solid, oval, capsulated mass of $2 \mathrm{x} 1 \mathrm{x}$ $1 \mathrm{~cm}$. The suspected diagnosis of schwannoma was confirmed by the final histopathological report, showing a protein s110 positivity at the immunohistochemistry staining.

No early or late complications after surgery were observed, and the patient was discharged after two days. During follow-up, flexible laryngoscopy performed after 1 month showed disappearance of the submucosal mass and no vocal fold motility impairment. The patient did not refer any change in her voice or other symptoms related to laryngeal mucosa sensitivity deficit like dysphagia (Figure 3 ).

\section{Discussion}

Laryngeal schwannomas are uncommon lesions and represent 0.1 to $1.5 \%$ of all benign laryngeal tumours. The vast majority of schwannomas occur as sporadical single lesions, affecting patients of all ages, but most commonly between the ages of 20 and 50, without
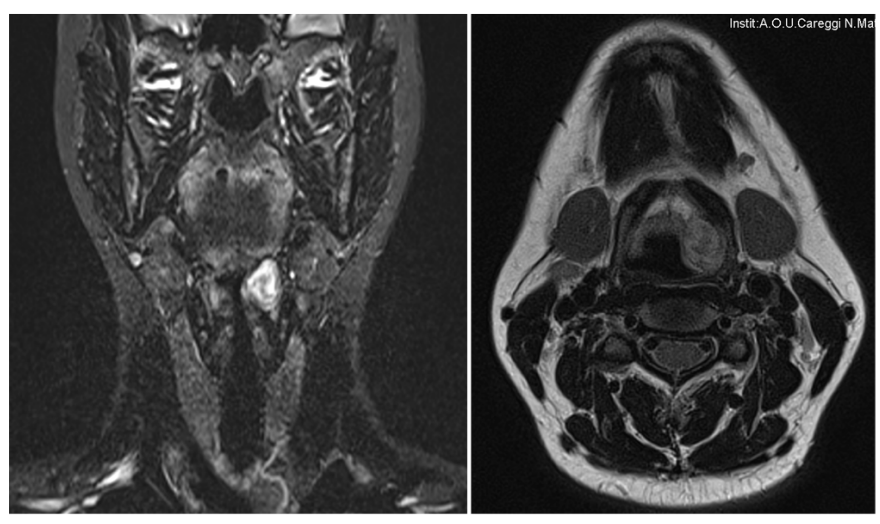

A

$\mathrm{B}$

Figure 1. Magnetic Resinante Imaging: (A) lesion appearing as hyperintense on T2 weighted image; (B) lesion appearing as hyperintense on T1 weighted image

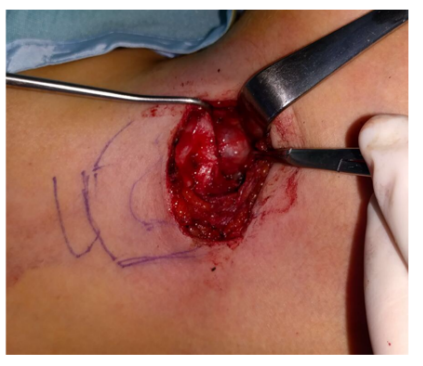

A

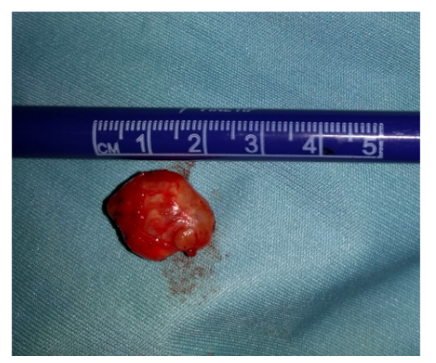

B
Figure 2. Surgical procedure and surgical specimen: (A) Excision of the lesion by blunt dissection.

Exposure of the surgical field by retracting the thyroid lamina by the hook. No cartilage incision

was needed; (B) solid, oval mass of $2.5 \mathrm{~cm}$ in its maximum diameter

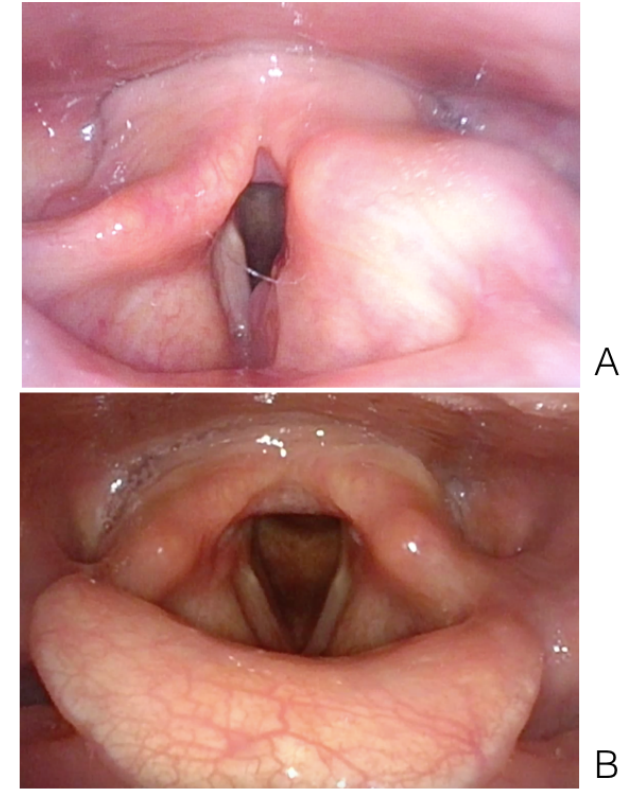

Figure 3. Laryngoscopy: (A) before surgical excision; (B) 1 month after the surgical excision

associated lifestyle risk factors [6]. In regards of gender predilection, no general agreement is observed in literature, with some authors reporting a female predominance [7].

Clinical presentation strictly depends on the location and size of the laryngeal lesion, and due to the slow-growing nature of schwannoma, most patients are asymptomatic until the tumour reaches dimension that determine a conflict with the surrounding structures and mass effect [4].

In a recent systematic review, Tulli et al. reported that the most common presentation was related to vocalisation, with hoarseness or dysphonia (71.2\%), followed by dysphagia (24.7\%), dyspnea $(23.3 \%)$ and foreign body sensation (16.4\%) [2].

On laryngoscopy, the characteristics finding for laryngeal schwannoma is a round, smooth submucosal swelling, usually involving the false vocal fold or aryepiglottic fold. Depending on the dimension, the lesion can obstruct the view of the true vocal fold, reduce the laryngeal inlet and caused an impairment in vocal cord mobility as a result of mass effect [1].

As for anatomical site, almost all neurogenic tumours of the larynx arise from the supraglottic area, but also the involvement of the true vocal folds, epiglottis, subglottis areas and postcricoid areas was described even though is extremely rare $[2,4]$.

With regards to diagnostic imaging methods, computer tomography (CT) and MRI are most frequently indicated.

CT scan shows a well-defined mass with heterogenic density on contrast enhancement, surrounded by a peripheral enhancing ring, and is useful to estimate the exact size and extent of the lesions. MRI generally offers a superior soft tissue delineation and provides more information on the cellularity of the lesion. Schwannomas have intermediate signal intensity in T1-weighted images and markedly increased signal intensity on $\mathrm{T} 2$-weighted images. The contrast enhancement observed after gadolinium tends to be uniform in small lesions, whereas heterogeneous in larger schwannomas [8]. 
In this clinical and radiological setting the main differential diagnosis that needs to be considered include non-neoplastic lesions (internal laryngocele, ectopic thyroglossal duct cyst, laryngeal cyst), and benign laryngeal tumours (neurofibroma, lipoma, adenoma, chondroma, paraganglioma, papilloma) $[2,4,9]$.

The definitive diagnosis of schwannoma requires histopathological examination. Enzinger and Weiss established the three histological criteria for the diagnosis: the presence of a capsule, the presence of Antoni A and or Antoni B areas and S-100 protein positivity. In Antoni A (cellular region), the spindle-shaped Schwann cells are compactly arranged with nuclei occasionally lining up in palisades to form Verocay bodies. Antoni B (less cellular) describe loosely arranged spindle Schwann cells within a myxoid matrix. S-100 protein is an acidic protein found in the supporting cells of the central and peripheral nervous system and the immunocytochemical stain for this protein acts as the main diagnostic tool for schwannoma. There are various methods for obtaining a histological diagnosis: fine needle aspiration cytology (FNAC), incisional biopsy and surgical excision. Previous reports indicate that FNAC is not recommended because is often inconclusive $[10,11]$. Whereas, review of literature reveals that incisional biopsy has higher diagnostic efficacy but presents several problems as the risk of insufficient material for diagnosis, formation of a scar that can complicate the following excision of the mass and overall the need to submit the patient to at least two surgical procedures for the complete excision of the lesion [2,9-11].

Surgical excision forms the mainstay of treatment for laryngeal schwannoma. Appropriate surgical therapy requires the complete excision of the mass avoiding injury to surrounding structures.

The surgical approach used depends on the basis of characteristics of the tumour (size and location) and the patient (endoscopic exposure). Generally, if the lesion is small and superficial, or originating from the true vocal folds the best choice is trans-oral endoscopic surgery either with conventional cold steel instruments or with laser. The trans-oral approach is a less-invasive procedure and provides several advantages as the absence of visible scar in the neck, fewer days of hospitalisation and no need to perform tracheotomy $[12,13]$.

For larger tumours, the external approach offers the best exposure of the surgical field facilitating the excision of the lesion without disruption of the capsule and damage of the overlaying mucosa. The most frequently used open approaches include medial thyrotomy (MT), lateral thyrotomy (LT), lateral pharingotomy and supra-thyroid alar cartilage approach (STACA) [2,4].

MT shows advantage in providing excellent exposure of the hemylarynx. However, to obtain optimal voice outcome, both cords must be placed exactly at the same height during the reapproximation of the cartilage [1]. To minimise the risk of injury to the vocal cord and laryngeal mucosa, some authors preferred LT procedure, that also provides a good exposure and good visibility of the paraglottic space $[10,14]$. Lateral pharingotomy approach is rarely used because of the possible risks of fistula and injury to nerves X and XII [4].

In 2015 Ueha et al. [15] described a novel extra laryngeal approach. With this procedure, laryngeal submucosal tumours can be removed easily without thyrotomy or any anatomical distortion to the laryngeal frameworks, accessing the paraglottic space by sectioning the sternohyoid and thyrohyoid muscles and the thyrohyod membrane of the affected side.

In our case, we performed an approach very similar to the STACA technique, but even less invasive by avoiding the disarticulation of the cricothyroid joint and the elective tracheotomy and by achieving the exposure of the submucosal mass without sectioning the thyrohyoid membrane along all the superior border of the alar cartilage.

After treatment, an appropriate follow-up for the detection of possible recurrence is needed, even if there is currently no consensus regarding the duration $[4,9]$.

\section{Conclusion}

Laryngeal schwannoma is a rare benign tumour with indolent growth pattern. Symptoms mainly have to be referred to the mass effects. Complete surgical excision is the treatment of choice and can be performed by either trans-oral or external approach depending on lesion dimension, patient's laryngeal exposure and surgeon's skills. We suggest that each case must be studied taking into account all the feasible options to provide a surgical approach tailored to the characteristics of the tumour and the patient.

\section{Acknowledgment}

Paper was presented as poster presentation at the 12th Congress of the European Laryngological Society (ELS) which has been held on May 16-19, 2018 at the Queen Elizabeth II Conference Centre in London.

\section{Statement of ethics}

The research was conducted ethically in accordance with the World Medical Association Declaration of Helsinki. The subject has given her written informed consent to publish her case (including publication of images).

\section{Disclosure statement}

The authors have no conflicts of interest to declare.

\section{Funding sources}

None to declare.

\section{Author contributions}

Each author has participated actively in designing and writing this article: Federica Martelli assisted in conception of the study, gave her important help by writing the manuscript and reviewing literature; Massimo Squadrelli assisted in surgical procedure, manuscript preparation and its discussion; Giacomo Spinato helped with literature review and the final draft review and approval; Giuditta Mannelli is the main creator of the work and critically reviewed and discussed the final manuscript.

\section{References}

1. Rosen FS, Pou AM, Quinn FB Jr (2002) Obstructive supraglottic schwannoma: a case report and review of the literature. Laryngoscope 112: 997-1002. [Crossref]

2. Tulli M, Bondi S, Smart CE, Giordano L, Trimarchi M, et al. (2018) Diagnosis and Treatment of Laryngeal Schwannoma: A Systematic Review. Otolaryngol Head Neck Surg 158: 222-231. [Crossref]

3. Ramakrishnan Y, Issing WJ (2011) Laryngeal schwannoma: case report and literature review. ISRN Otolaryngol 2011: 540643. [Crossref]

4. Wong BLK, Bathala S, Grant D (2017) Laryngeal schwannoma: a systematic review. Eur Arch Otorhinolaryngol 274: 25-34. [Crossref]

5. Piazza C, Mangili S, Bon FD, Paderno A, Grazioli P, et al. (2014) Preoperative clinical predictors of difficult laryngeal exposure for microlaryngoscopy: the Laryngoscore. Laryngoscope 124: 2561-2567. [Crossref]

6. Biswas D, Marnane CN, Mal R, Baldwin D (2007) Extracranial head and neck schwannomas--a 10-year review. Auris Nasus Larynx 34: 353-359. [Crossref] 
7. Romak JJ, Neel HB 3rd, Ekbom DC (2017) Laryngeal Schwannoma: A Case Presentation and Review of the Mayo Clinic Experience. J Voice 31: 129. [Crossref]

8. Ebmeyer J, Reineke U, Gehl HB, Hamberger U, Mlynski R, et al. (2009) Schwannoma of the larynx. Head Neck Oncol 1: 24. [Crossref]

9. Tulli M, Bondi S, Bussi M (2017) Voluminous laryngeal schwannoma excision with a mini-invasive external approach: a case report. Acta Otorhinolaryngol Ital 37: 242 244. [Crossref]

10. Cohen S, Sinacori JT, Courey MS (2004) Laryngeal schwannoma: diagnosis and management. Otolaryngol Head Neck Surg 130: 363-365. [Crossref]

11. Zhang H, Cai C, Wang S, Liu H, Ye Y, et al. (2007) Extracranial head and neck schwannomas: a clinical analysis of 33 patients. Laryngoscope 117: 278-281. [Crossref]
12. Wang B, Dong P, Shen B (2014) Laryngeal schwannoma excised under a microlaryngoscope without tracheotomy: A case report. Exp Ther Med 7: 1020-1022.

13. Xu J, Zheng Y, Li G, Su X (2012) A rare finding of multiple schwannomas in the epiglottis. Otolaryngol Head Neck Surg 147: 1160-1161. [Crossref]

14. Thomé R, Thomé DC, De La Cortina RA, Lateral thyrotomy approach on the paraglottic space for laryngocele resection. Laryngoscope 110: 447-450.

15. Ueha R, Nito T, Sakamoto T, Fujimaki Y, Yamauchi A, et al. (2015) Supra-thyroid alar cartilage approach for the complete resection of laryngeal submucosal tumors and postoperative voice quality. Eur Arch Otorhinolaryngol 272: 2907-2913. [Crossref]

Copyright: (C2019 Martelli F. This is an open-access article distributed under the terms of the Creative Commons Attribution License, which permits unrestricted use, distribution, and reproduction in any medium, provided the original author and source are credited. 